Copyright by the American Institute of Physics (AIP). Singh, G; Saraf, RF; Martin, Y., "Solid-like dynamics in ultrathin films

of polymeric liquids," Appl. Phys. Lett. 83, 5410 (2003); http://dx.doi.org/10.1063/1.1637145

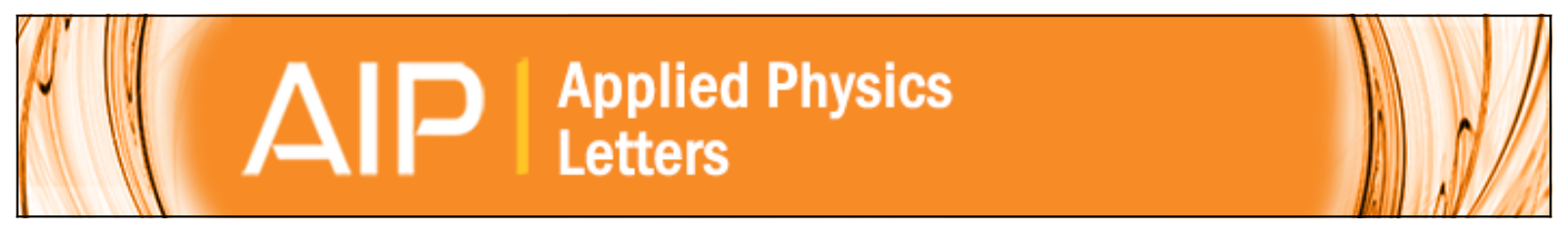

\title{
Solid-like dynamics in ultrathin films of polymeric liquids
}

Gaurav Singh, Ravi F. Saraf, and Yves Martin

Citation: Applied Physics Letters 83, 5410 (2003); doi: 10.1063/1.1637145

View online: http://dx.doi.org/10.1063/1.1637145

View Table of Contents: http://scitation.aip.org/content/aip/journal/apl/83/26?ver=pdfcov

Published by the AIP Publishing

Over 700 papers \&

presentations on

multiphysics simulation vew now

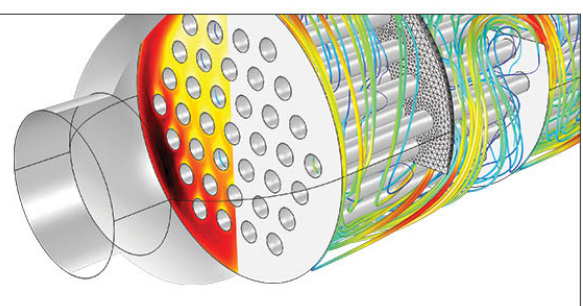




\title{
Solid-like dynamics in ultrathin films of polymeric liquids
}

\author{
Gaurav Singh and Ravi F. Saraf ${ }^{a)}$ \\ Department of Chemical Engineering, Virginia Tech, Blacksburg, Virginia 24061 \\ Yves Martin \\ T.J. Watson Research Centre, IBM Corporation, Yorktown Heights, New York 10598
}

(Received 11 April 2003; accepted 6 November 2003)

\begin{abstract}
In this letter, we demonstrate that, at mesoscales, nonferroelectric liquid films of poly(dimethyl siloxane) exhibit significant electrostriction not present in the corresponding bulk state. Remarkably, the observed electrostrictive effect has a response time $<20 \mu \mathrm{s}$ in contrast to $>5 \mathrm{~ms}$ recorded in conventional bulk (ferroelectric) polymers. The emergence of this fast electrostrictive strain in thin films is explained in terms of the amalgamation of two contrasting dynamic features - the influence of a highly mobile, viscous layer (at the air/film interface) on the less-mobile, but fast responding, solid-like layer at the film/substrate interface. The effect is observed for thickness below $200 \mathrm{~nm}$.

(C) 2003 American Institute of Physics. [DOI: 10.1063/1.1637145]
\end{abstract}

The interfacial structure and properties of soft matter, such as polymers, deviate significantly from the bulk. It is recognized that molecular segments of polymers closer to a "free" surface (i.e., film/air interface) have higher mobility than the bulk. This is beautifully illustrated by orienting molecular chains at the surface by simply rubbing the surface of a vitrified (or crystalline) polymer with a soft material. ${ }^{1-3}$ The polymer molecular segments at the film/substrate interface, in contrast to the bulk, experience stronger dispersive forces from the substrate molecules that are more polarizable and/or packed denser. This additional interaction may be modeled as the "tail end" of a van der Waals long-range interaction emanating from the substrate. ${ }^{4}$ These stronger interactions lead to densification at the film/substrate interface. ${ }^{5,6}$ The densification effect is shown to manifest as a solid-like elastic behavior in $<1 \mathrm{~Hz}$ dynamic strain experiments by surface force apparatus. ${ }^{7}$

In this letter, we describe a study, where fast dynamics, at $>10 \mathrm{kHz}$, in thin films of polymeric liquids is observed. The dynamic behavior is obtained by deforming the film under oscillating electric field and measuring the electrostrictive strain by the interferometric method. The results indicate elastic-like dynamics with a strong dependence on film thickness. The fast dynamic behavior fades away at a film thickness above $200 \mathrm{~nm}$. The observation is explained by considering a non-Gibbs film where the two interfaces commence to overlap, such that the structure of the intermediate core layer is different from the bulk. ${ }^{8}$

A $2000 \mathrm{~g} / \mathrm{mol}$ poly(dimethyl siloxane) (PDMS) film is spin casted from hexane solution on a $\mathrm{SiO}_{2} / \mathrm{Si}$ wafer with Au electrodes at $30 \mu \mathrm{m}$ spacing. The radius of gyration $R_{g}$ is $\sim 6.5 \times 10^{-2} \mathrm{M}^{0.5} \mathrm{~nm} \sim 3 \mathrm{~nm}^{.}{ }^{9}$ The wafer is treated in $\mathrm{H}_{2} \mathrm{SO}_{4} / \mathrm{H}_{2} \mathrm{O}_{2}$ (3:1) solution to produce hydroxyl, Si-OH groups at the surface prior to spin casting. ${ }^{10}$ The PDMS is wetting on the surface. The thicknesses of the films are measured by a spectroscopic ellipsometer. The absence of interference fringes at the electrode edge under the Nomarski microscope indicates a negligible meniscus contribution in

a)Electronic mail: rsaraf@vt.edu the film between the $30 \mu \mathrm{m}$ electrode spacing. An ac electric field, $E(\omega)$, at a frequency $\omega$, is applied via the electrodes. The field, parallel to the film surface, stretches the film parallel to the substrate. The relative angle between the polarization of light and the electric field does not affect the signal. As a result, it shrinks along the thickness direction due to the Poisson effect. The amplitude of thickness modulation, $\Delta d$ is measured by the differential interferometer setup shown in Fig. 1(a). Interference between phase-modulated "reference" and "sample" beams is measured at the detector. The reference beam is phase modulated at $80 \mathrm{MHz}$ by a Bragg cell. The sample beam is phase modulated at $2 \omega$ due to a change in the optical path length caused by (secondorder) oscillation in the sample thickness due to the electrostriction effect from the applied field, $E(\omega)$. Figure 1(b) shows the typical signal observed in the spectrum analyzer. The central peak at $80 \mathrm{MHz}$ is due to the carrier frequency from the Bragg cell. The two satellite peaks at $80 \mathrm{MHz} \pm 2 \omega$ are due to the phase modulation from the sample. Frequency below $8 \mathrm{kHz}$ is too close to the background from the main peak, and therefore constitutes the lower limit of our measurement. The absence of satellite peaks at $80 \mathrm{MHz} \pm \omega$ indicates that there are no (linear) piezoelectric effects caused by dipole orientation. This is expected, since PDMS is a nonpolar fluid. From the power ratio of the main peak and the (second-order) satellite peak, $R$, the change in optical path length $\Delta(n d)=R \lambda / \pi$ is obtained, where $n$ is the refractive index of the film. ${ }^{11}$ We note that the intensity change (i.e., amplitude modulation) due to the change in reflectivity from the sample due to electric field is well within the noise.

The strain amplitude, $\epsilon=\Delta d / d_{0}<0$. For $\epsilon<10^{-3} \%$ (see Fig. 2), with respect to the undeformed state $\left(n_{0}, d_{0}\right)$, $\Delta(n d)=n_{0} \Delta d+d_{0} \Delta n$. The $\Delta n$ increases due to two primary reasons: (i) increase in density, $\rho$, caused by the thickness reduction $\left(\Delta \rho \sim-\Delta d / d_{0}^{2}\right)$, and (ii) orientation of the induced dipole along the field direction. For small strain, in PDMS, it is estimated that by correcting $n$ by $n-0.1$, the effect of refractive index change (i.e., $d_{0} \Delta n$ ) can be accounted for as $\Delta(n d)=\left(n_{0}-0.1\right) \Delta d .{ }^{12}$ For convenience, we report our result as strain magnitude, $|\epsilon|$. 


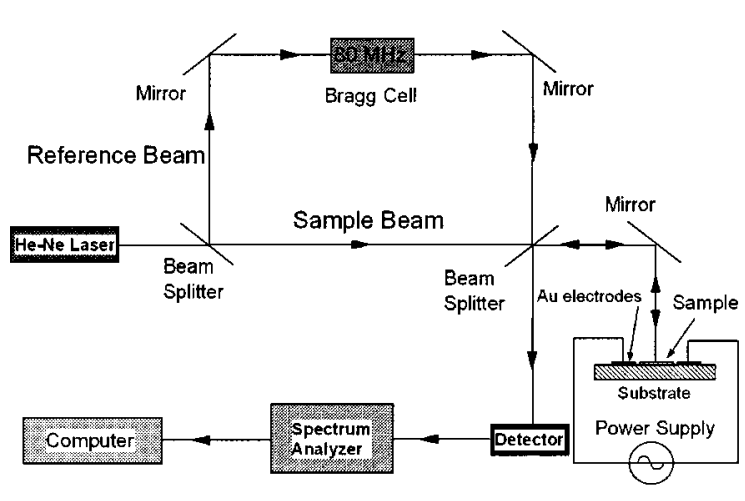

(a)

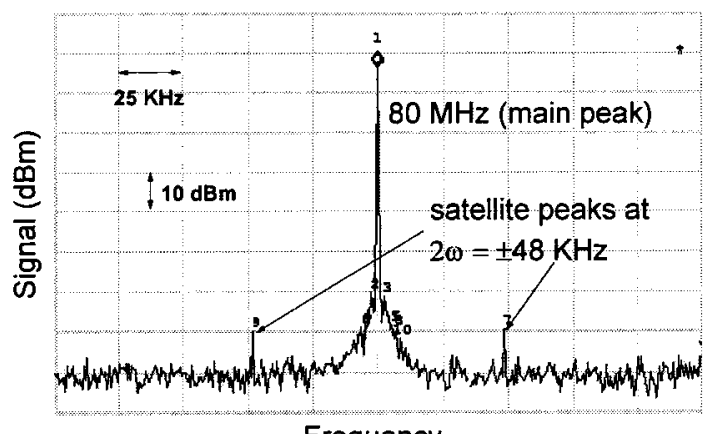

(b)

FIG. 1. (a) Differential interferometric setup to measure the amplitude of thickness modulation, $\Delta d$ due to electric field $E e^{-i \omega t}$. The interference amplitude measured at the detector is amplified and recorded in a spectrum analyzer connected to a computer for data acquisition. (b) Raw data: the typical power spectrum of the signal in the spectrum analyzer shows the central $80 \mathrm{MHz}$ main peak (No. 1) due to the Bragg cell and two -80.08 and $-79.28 \mathrm{dBm}$ satellite peaks (Nos. 9 and 7) at $80 \mathrm{MHz} \pm 2 \omega$.

Figure 2 shows the dynamic response of thin films as a function of frequency. The fast response in the $10-64 \mathrm{kHz}$ or $100-16 \mu$ s range indicates that the electrostrictive dynamics is primarily elastic. Since the polymer is $\sim 148{ }^{\circ} \mathrm{C}$ above its bulk $T_{g}$, the film should primarily be liquid except for the "solid-like" layer in the vicinity of the film/substrate inter-

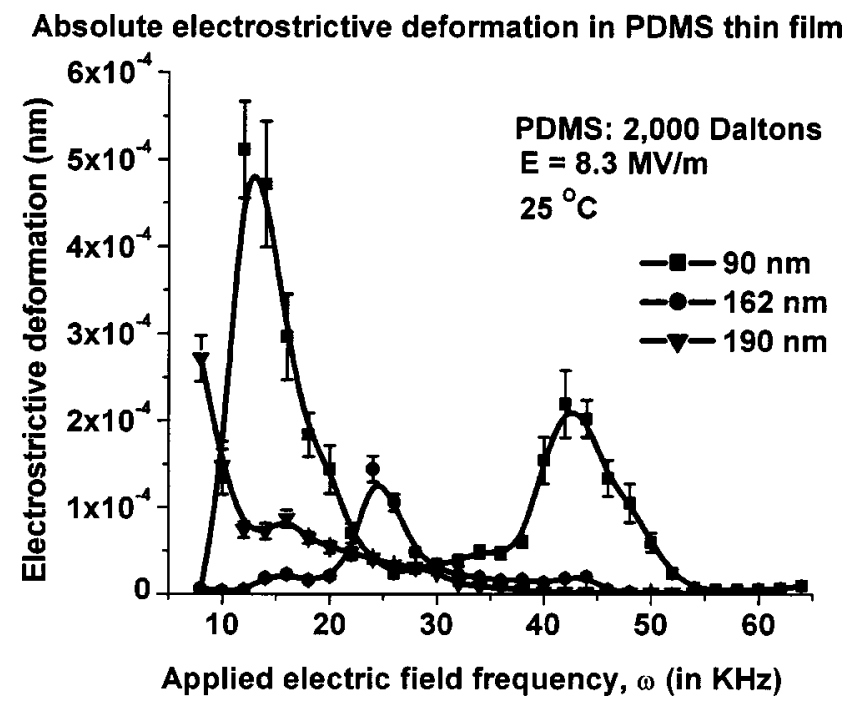

FIG. 2. $\omega$ vs $\Delta d$ response of the PDMS film at the thickness and electric field amplitude indicated. The amplitude of the thickness modulation, $\Delta d$, instead of $\epsilon$ is plotted for better visualization. The error bars are standard deviation based on 30 data points taken at $0.1 \mathrm{~s}$ intervals. face. The solid-like layer formation is observed under slow $(<1 \mathrm{~Hz})$ dynamic mechanical experiments in the polymer thin film confined between two solid surfaces. ${ }^{7,13}$ The presence of such an interfacial elastic layer for PDMS on the silica surface is confirmed using quasielastic neutron scattering and nuclear magnetic resonance. ${ }^{14,15}$ If the fast, viscoelastic response were exclusively due to the solid-like film/substrate interfacial layer, the $\Delta d$ measured in Fig. 2 should be independent of $d_{0}$, except for inertial damping from the top layer. However, the significant change in the relaxation peaks both in terms of frequency position and intensity suggests the systematic inertial damping effects do not explain the observation.

The significant thickness effects suggests that the film cannot be considered a simple Gibbs layer where the excess thermodynamic properties are the sum of the air/film interface (top layer), bulk (middle layer), and film/substrate interface (bottom layer). In the non-Gibbs thin film, the top and bottom layers overlap to form a modified middle layer that is different compared to the bulk of a conventional Gibbs film. ${ }^{8}$ An alternate description would be that the bulk layer is influenced by the long-range van der Waals interaction due to the dense, highly polarizable substrate, leading to disjoining pressure (П) in the overlap region. ${ }^{4}$

The $\Pi$ may alter the effective electromechanical response as follows. Neglecting gravitational effects and assuming the long-range attraction to be simply the van der Waals interaction tail $\sim 1 / d^{m}$, (where $\mathrm{m}=2$ for nonretarded case), the disjoining pressure $\Pi \sim A /(6 \pi \mathrm{d}) .{ }^{16}$ Where, $A$ $(>0)$ is the difference in the Hamaker constant for the substrate-liquid and liquid-liquid interactions. The $\Pi>0$ implies that the disjoining pressure is acting opposite to hydrostatic pressure and tends to thicken the film. Thus, due to $\Pi$, the film will have a "bounce-back" effect as the electric field decreases the thickness. For small deformation (as is the case with $\epsilon<10^{-3} \%$ ) the restoring force is $\sim 1 / d^{2}$. Thus, a faster response is expected due to $\Pi$ in thinner films. This is consistent with Fig. 2, where in the high-frequency part of the spectra, more activity is observed for thinner films.

The response likely from the middle, overlap layer, as mentioned above, is influenced by $\Pi$. Analogous to the dynamic mechanical behavior, ${ }^{17}$ the "relaxation" peaks suggest that the behavior is viscoelastic, which may be considered an amalgamation of the high-mobility and viscous top interface, and the fast-responsive and elastic bottom interface.

Interestingly, the dynamic character of the overlap region does not change, as seen by the relatively invariant relaxation peaks at 14,24 , and $44 \mathrm{kHz}$. However, the relative magnitude changes significantly with thickness. One way to interpret the observation is by considering the intermediate layer as a "two-phase" structure composed of a more ordered mesomorphic structure that is elastic, and the "constrained" amorphous structure that is viscous and less elastic. The $90 \mathrm{~nm}$ film presumably is mostly mesomorphic with essentially the two relaxation peaks at 44 and $14 \mathrm{kHz}$. As the thickness increases, the overlap decreases making the structure more disordered. Thus, the $162 \mathrm{~nm}$ film has a more "constrained" amorphous phase giving rise to a strong 24 $\mathrm{kHz}$ peak (absent in the $90 \mathrm{~nm}$ film) and a small amount of a mesomorphic phase, as seen by the weak relaxation peaks/ 


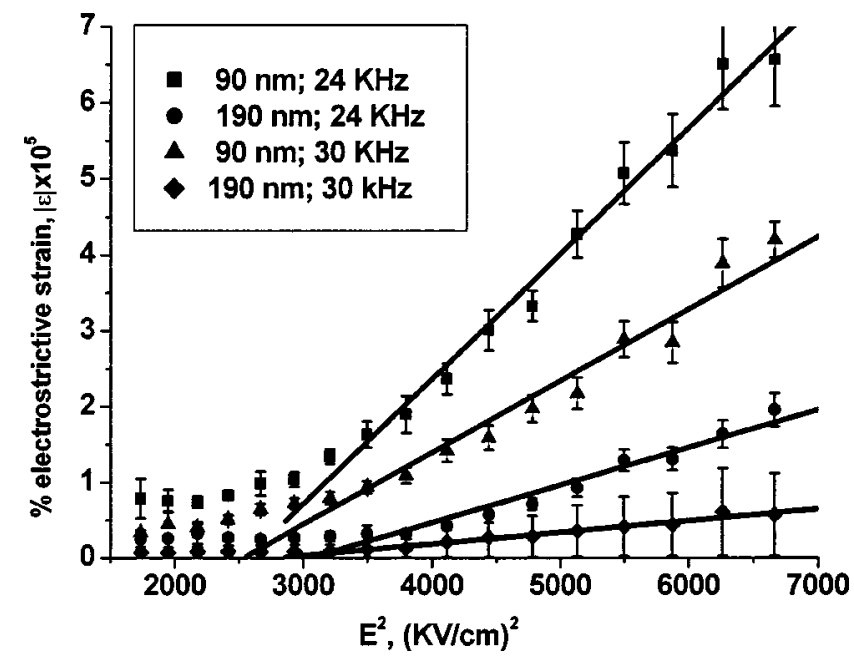

FIG. 3. Linear behavior fitness (including the error bars) range from 0.98 to 0.99 . The slopes, $\nu \beta$ in descending order are $165,95,50$, and $15(\mathrm{pm} / \mathrm{V})^{2}$. This is expectedly small. However, the $\beta$ is significant, considering no electrostrictive response is reported for bulk films.

shoulders at 14 and $44 \mathrm{kHz}$. Eventually, at $190 \mathrm{~nm}$, the film is closer to the classical Gibbs layer where the overlap region is small, as manifested by weak peaks due to the mesomorphic $(14 \mathrm{kHz})$ and constrained amorphous $(24 \mathrm{kHz})$ phases. Beyond $200 \mathrm{~nm}$, no electrostriction is observed, indicating that the top interface (and the bulk) is too slow (i.e., viscous), and the elastic bottom interface is too rigid (i.e., low mobility) or too thin.

The electrostriction coefficient, $\beta$ (a tensor) is defined as the proportionality constant between the strain, $\epsilon$, and $E^{2}$. For isotropic system with uniaxial $E, \beta$ is a scalar quantity. In our study, $E$ is parallel to the film plane and the strain is measured along the thickness plain [see Fig. 1(a)]. Thus, $\epsilon$ $=\Delta d / d_{0}=\nu \beta E^{2}$, where $\nu$ is the Poisson ratio of the polymer. Figure 3 shows a typical $|\epsilon|$ vs $E^{2}$ behavior in the relatively "flat" portion of the frequency response (see Fig. 2). The (expected) linearity is observed, but beyond a threshold $E$ of $\sim 53 \mathrm{kV} / \mathrm{cm}$. The threshold field cannot be attributed to the experimental measurement limit since the lines do not converge at $(0,0)$ but $\left[\sim 2800(\mathrm{kV} / \mathrm{cm})^{2}, 0\right]$. A possible explanation may be an activation barrier to commence the long-range segmental motion. For example, at small fields, small polarization may cause only local orientation, while at large fields significant polarization may lead to (cooperative) long-range chain orientation causing larger deformation, and hence, larger $\beta$.

One of the authors (R.F.S.) thanks the Office of Naval Research (Grant No. N00014-01-1-0977), and Jeffress Memorial Trust, VA, for financial support; and Jayan Krishnaswami for data acquisition automation.

${ }^{1}$ J. C. Wittmann and P. Smith, Nature (London) 352, 414 (1991).

${ }^{2}$ M. F. Tony, T. P. Russell, J. A. Logan, H. Kikuchi, J. M. Sands, and S. K. Kumar, Nature (London) 374, 709 (1995).

${ }^{3}$ A. M. Higgins and R. A. L. Jones, Nature (London) 404, 476 (2000).

${ }^{4}$ P. G. de Gennes, Rev. Mod. Phys. 57, 827 (1985).

${ }^{5}$ D. N. Theodorou, Physics of Polymer Surfaces and Interfaces (Butterworth-Heinemann, Boston, MA, 1992), p. 139.

${ }^{6}$ S. K. Kumar, M. Vacatello, and D. Y. Yoon, Macromolecules 23, 2189 (1990).

${ }^{7}$ S. Granick, Science 258, 1374 (1991).

${ }^{8}$ B. V. Derjaguin, N. V. Churaev, and V. M. Muller, Surface Forces (Consultants Bureau, New York, 1987), p. 26.

${ }^{9}$ Polymer Handbook, 2nd ed., edited by J. Brandrup and E. H. Immergut (Wiley, New York, 1975), p. IV-48.

${ }^{10}$ G. Evmenenko, S. W. Dugan, J. Kmetko, and P. Durra, Langmuir 17, 4021 (2001)

${ }^{11}$ P. G. Charlette, lan. W. Hunter, and C. J. H. Brenan, Rev. Sci. Instrum. 63, 241 (1992).

${ }^{12} \Delta n d=\left[n-\left(N_{A v} \alpha \rho / 3 \epsilon_{0} M\right)\left[\left(n^{2}+2\right)^{2} / 6 n\right]-S G\right] \Delta d$, where the first term is from $n \Delta d$, the second term is from densification, and the third term due to orientation is simply stress optical coefficient $S$ time modulus $G$. Where $\rho \sim 1 \mathrm{~g} / \mathrm{cm}^{3}$, electronic polarizability, $\alpha \sim 5.08 \times 10^{-39} \mathrm{C}^{2} \mathrm{~m}^{2} / \mathrm{J}$, $M=2000$ Daltons, $n \sim 1.45$, estimated $S \sim 3.7 \times 10^{-11} \mathrm{~m}^{2} / \mathrm{N}$ (based on Ref. 7, pp. 380 and 382), and G $\sim 1 \mathrm{MPa}$. Thus, the third term $\sim 10^{-5}$ is negligible compared to second term of $\sim 0.1$. Thus, the correction is simply, $\Delta n d=\left(n_{0}-0.1\right) \Delta d$, where the correction to $n_{0}$ is independent of $d$.

${ }^{13}$ H.-W. Hu and S. Granick, Science 258, 1339 (1992).

${ }^{14}$ V. Arrighi, J. S. Higgins, A. H. Burgess, and G. Floudas, Polymer 39, 6369 (1998).

${ }^{15}$ V. M. Litvinov, H. Barthel, and J. Weis, Macromolecules 35, 4356 (2002).

${ }^{16}$ F. Brochard Wyart and J. Daillant, Can. J. Phys. 68, 1084 (1990).

${ }^{17}$ J. D. Ferry, Viscoelastic Properties of Polymers, 3rd ed. (Wiley, New York, 1980) 